\title{
Mechanism of regulation of stem cell differentiation by matrix stiffness
}

\author{
Hongwei LV ${ }^{1,2}$, Lisha Li ${ }^{1 *}$, Meiyu Sun ${ }^{1}$, Yin Zhang ${ }^{1,2}$, Li Chen ${ }^{1,2}$, Yue Rong ${ }^{1,3}$ and Yulin Li ${ }^{1 *}$
}

\begin{abstract}
Stem cell behaviors are regulated by multiple microenvironmental cues. As an external signal, mechanical stiffness of the extracellular matrix is capable of governing stem cell fate determination, but how this biophysical cue is translated into intracellular signaling remains elusive. Here, we elucidate mechanisms by which stem cells respond to microenvironmental stiffness through the dynamics of the cytoskeletal network, leading to changes in gene expression via biophysical transduction signaling pathways in two-dimensional culture. Furthermore, a putative rapid shift from original mechanosensing to de novo cell-derived matrix sensing in more physiologically relevant three-dimensional culture is pointed out. A comprehensive understanding of stem cell responses to this stimulus is essential for designing biomaterials that mimic the physiological environment and advancing stem cell-based clinical applications for tissue engineering.
\end{abstract}

\section{Introduction}

Stem cells hold enormous potential for treating a broad spectrum of human diseases due to their multipotency [1-3]. Besides biochemical signals, stem cell maintenance and differentiation are regulated by biophysical aspects of the microenvironment, including mechanical loading, substrate material property and cell shape $[4,5]$. With the development of biomimetic substrates, new data continue to reveal more and more inspiring details of extracellular matrix (ECM) stiffness, which profoundly impacts on stem cell self-renewal and commitment [6-8]. ECM stiffness is usually represented by the elastic modulus or Young's modulus. Generally, ECM stiffness, which matches the stiffness of native tissue, guides stem

\footnotetext{
* Correspondence: lilisha@jlu.edu.cn; ylli@jlu.edu.cn

${ }^{1}$ The Key Laboratory of Pathobiology, Ministry of Education, Jilin University, Changchun 130021, China

Full list of author information is available at the end of the article
}

cell differentiation down corresponding tissue lineages. For instance, substrates approximating to the elastic moduli of brain $(0.1$ to $1 \mathrm{kPa})$, pancreas $(1.2 \mathrm{kPa})$, cartilage $(3 \mathrm{kPa})$, muscle $(8$ to $17 \mathrm{kPa})$ and bone tissue (25 to $40 \mathrm{kPa})$ direct stem cells, especially mesenchymal stem cells (MSCs), to commit to neurocytes, beta cells, chondrocytes, myoblasts and osteoblasts, respectively $[6,9,10]$. There is a growing interest in understanding how stem cells feel and respond to specific stiffness; how the mechanical cue is converted into intracellular signaling cascades; and how gene expression changes and stem cell fate are determined.

We summarize the mechanotransduction steps activated by matrix stiffness in stem cell differentiation (Figure 1). Based on recent experiments in two-dimensional models, the mechanism of mechanotransduction of stem cells is probably associated with the integrin-cytoskeletal-based feedback loop between mechanical signals and biochemical signals in the background of the signaling network to determine their fates. Different mechanisms of matrix stiffness mechanotransduction may exist in three-dimensional environments, which are more physiologically relevant.

\section{Integrin as the starting point for mechanosensing}

The mechanical link between the ECM and the cytoskeleton are large protein assembly complexes known as focal adhesions, which involve integrins as major adhesion receptors [11]. Adapter proteins, including talin and vinculin, connect the cytoskeleton with integrins. Integrins are heterodimers comprising $\alpha$ and $\beta$ subunits, each with multiple types. The binding affinity and specificity of these subunits for various ECM proteins are different, and they also play different roles in regulating the response of stem cells to mechanical properties of the microenvironment. For instance, $\beta 3$ integrin mediates MSC myogenic differentiation induced by substrates with medium stiffness [12], while $\alpha 2$ integrin modulates osteogenesis of MSCs on stiff matrix [13]. As a vital cell surface transmembrane receptor, integrin mediates focal adhesion assembly, cytoskeletal organization, and a 


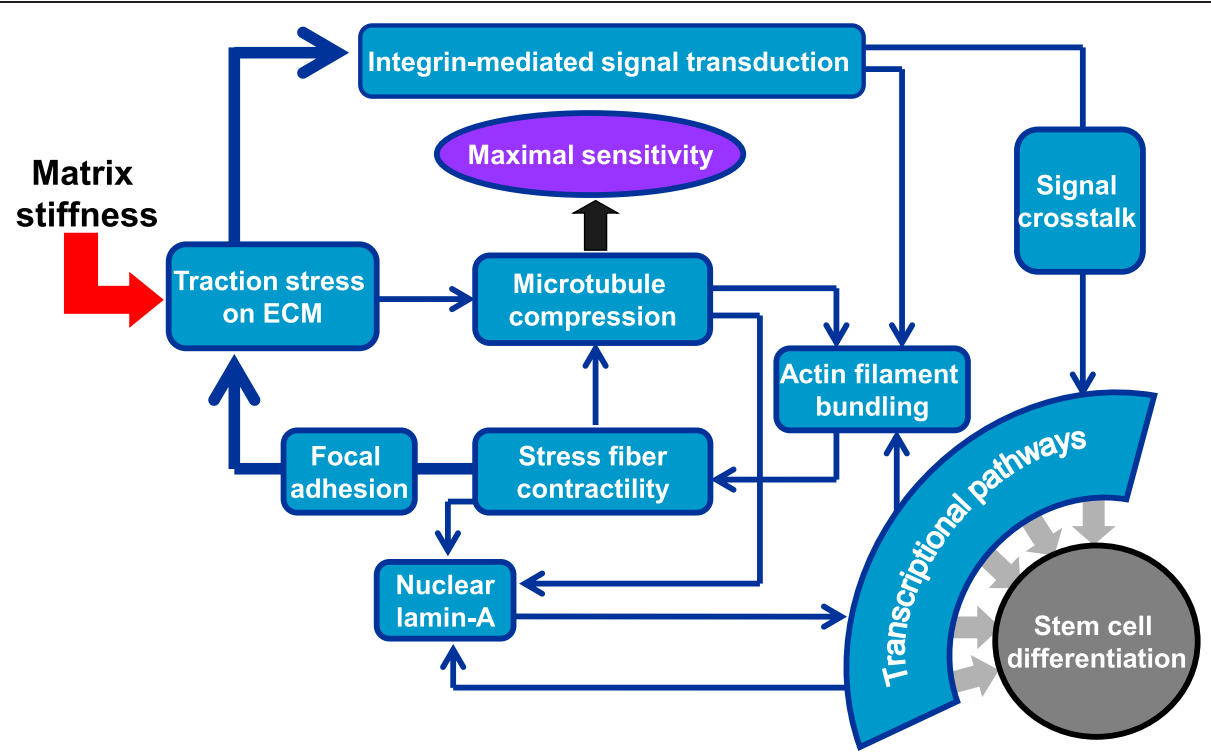

Figure 1 Mechanism of mechanotransduction of stem cells activated by matrix stiffness. The initial tension caused by stress fiber contraction is balanced by the microtubules resisting the resulting compression forces and the traction stress exerted on the extracellular matrix (ECM) across the focal adhesions, which directly cause the resultant force determined by matrix stiffness, contributing to microtubule compression. Then, the cell reads out the resultant forces from traction stress through the activation of integrin-mediated signal transduction pathways, which mediate actin filament polymerization and therefore change stress fiber contractility. Also, the initial tension from stress fiber contraction and the opposing compressive forces exerted by microtubules might also transmit into the nucleus and be resisted by lamin-A, which in turn promotes cell contractility by activating the transcriptional pathway that regulates actin filament bundling. Through cytoskeleton-based feedback loops, a cell changes its maximal mechanosensitivity close to the microtubule compression determined by matrix stiffness. Some transcriptional pathway modulates lamin-A expression, and feedback by lamin-A indirectly regulates transcriptional pathways, which crosstalk with integrin-mediated signaling and ultimately direct stem cell differentiation.

cascade of downstream signal transduction events through its activation during mechanotransduction [14]. Importantly, integrin transmits signals bidirectionally; that is, integrin not only transmits outside-in signals from the extracellular environment, but also passes intracellular stimuli to the outside of the cells [15]. The intracellular stimuli induce talin and kindlin to bind to the cytoplasmic domains of integrin $\beta$ subunits, which activates the binding of integrins and ligands [16]. A growing number of studies suggest that mechanical stiffness modulates the self-renewal and differentiation of stem cells through integrin-mediated transduction pathways $[13,17]$.

A host of intracellular signaling proteins are tightly associated with focal adhesions, such as focal adhesion kinase (FAK), a key component of mechanosensing. Under mechanical stimulation, the conformation of these signaling molecules may change, exposing phosphorylation sites and leading to activation of kinase cascades, transport of intracellular signaling molecules and changes in gene expression [18,19]. Among these signaling molecules, integrins are essential for mechanical signal perception, and are orientated at the start of the process of mechanotransduction induced by matrix stiffness [20].

\section{Integrin downstream signaling pathways}

Integrin signaling interplays directly or indirectly with other pathways during mechanotransduction, including those involving RhoA, bone morphogenetic protein (BMP)/Smad and FAK. $\beta 3$ integrin activity and RhoA signaling mediate the generation of cytoskeleton tension [12]. MSCs on micropatterned ECM with medium stiffness $(10.2 \mathrm{kPa})$ preferentially recruit $\beta 3$ integrin to develop larger and elongated focal adhesion complexes, which subsequently activate RhoA signaling [12]. It is known that RhoA activity is regulated by a wealth of GTP exchange factors or GTPase activating proteins (GAPs), including p190RhoGAP. The activation of RhoA leads to the stimulation of the contraction indicator myosin light chain kinase (MLCK), which cooperates with acto-myosin and actin filaments to promote stress fiber assembly to generate the appropriate cytoskeleton tension [21]. Additionally, $\beta 1$ integrin internalization inhibits the $\mathrm{BMP} /$ Smad signaling pathway [17]. Soft substrates lead to a substantial increase in the active form of $\beta 1$ integrin and a reduction in the cell surface distribution of $\beta 1$ integrin in MSCs by enhancing the detachment of integrin-ECM protein complexes [17]. In turn, the detachment drives activated integrin internalization through caveolae/raftdependent endocytosis. The enhanced uptake of $\beta 1$ 
integrin subsequently influences membrane localization of BMP receptor (BMPR) by promoting BMPR endocytosis. This endocytosis prevents BMPR from binding to ligands and thus inhibits the phosphorylation of downstream Smad 1/5/8 [17]. Furthermore, the state switches in $\alpha 5 \beta 1$ integrin link the cytoskeleton to FAK pathways [20]. FAK is a fundamental signaling molecule in the integrin-mediated signal transduction pathway [22]. During the mechanosensing process, myosin IImodulated cytoskeletal tension triggers the $\alpha 5 \beta 1$ integrin to switch from a relaxed state to a tensioned state. The switch in integrin state directly monitors the $\alpha 5 \beta 1$ fibronectin bond strength via engaging the synergy site in fibronectin, and links the cytoskeleton to FAK pathways [20]. FAK signaling in turn promotes activation of integrin, leading to enhanced cell adhesion on ECM [23].

\section{Cytoskeletal-based feedback loops to reach maximal mechanosensitivity}

Various studies have demonstrated that substrate stiffnessdriven lineage specification of stem cells changes when actomyosin contractility is blocked or increased by pharmacological cytoskeletal inhibitors in two-dimensional culture
[6,24-26] (Table 1). In other words, actomyosin contractility is critical for cells to perceive the stiffness of the microenvironment. Actomyosin contractility is modulated by actomyosin or myosin through sliding actin filaments in a polar fashion [27], and actomyosin filaments, known as stress fibers, mediate signal transduction from the ECM through integrins [28]. The response of the contraction rate or the contractile traction force results specifically from substrate stiffness and not cell tension or height. In an atomic force microscopy experiment [29], the cellular contraction rate, which represents the change in contractile traction force per unit time $(\mathrm{dF} / \mathrm{dt})$, rapidly increased while the corresponding contraction velocity, which is the change in cell height per unit time $(\mathrm{dx} / \mathrm{dt})$, decreased in response to increases in matrix stiffness. Focal adhesions, which represent dynamic actin-integrin linkage, provide essential ways of mechanical transmission of stiffness sensing through the contractility of stress fibers at the early stage of differentiation. Vinculin, a component of focal adhesion complexes, displays a more punctate structure on stiff substrates, but blocking of integrin-ECM binding by arginine-glycine-aspartate-serine (RGDS) leads to more diffuse vinculins and inhibits MSC osteogenesis

Table 1 Effects of pharmacological cytoskeletal inhibitors on stem cell differentiation induced by matrix stiffness

\begin{tabular}{|c|c|c|c|c|}
\hline Chemical & Binding target & Mechanism & $\begin{array}{l}\text { Stem cell } \\
\text { types }\end{array}$ & Effects on differentiation \\
\hline \multirow[t]{3}{*}{ Blebbistatin } & Myosin ATPase & $\begin{array}{l}\text { Inhibits actomyosin contractility by } \\
\text { blocking non-muscle myosin II ATPase } \\
\text { activity }\end{array}$ & MSCs & $\begin{array}{l}\text { Two-dimensional: blocks neurogenesis at } 0.1 \text { to } 1 \\
\mathrm{kPa} \text {, myogenesis at } 8 \text { to } 17 \mathrm{kPa} \text {, osteogenesis at } 25 \\
\text { to } 40 \mathrm{kPa}[6] \text {; suppresses chondrogenesis at } 1 \mathrm{kPa} \\
\text { [25]; inhibits osteogenesis at } 47.5 \mathrm{kPa} \text { [24] }\end{array}$ \\
\hline & & & $\begin{array}{l}\text { Mammary } \\
\text { progenitor cells }\end{array}$ & $\begin{array}{l}\text { Two-dimensional: abrogates myoepithelial cell } \\
\text { differentiation at } 4 \mathrm{kPa} \text { [60] }\end{array}$ \\
\hline & & & MSCs & $\begin{array}{l}\text { Three-dimensional: no obvious effect on hypertrophic } \\
\text { differentiation at approximately } 53.6 \mathrm{kPa} \text { [48]; no } \\
\text { obvious effect on osteogenesis at } 0.2 \text { to } 59 \mathrm{kPa}[46]\end{array}$ \\
\hline \multirow[t]{2}{*}{ ML-7 } & $\begin{array}{l}\text { Myosin light chain } \\
\text { kinase }\end{array}$ & $\begin{array}{l}\text { Inhibits actomyosin contractility by } \\
\text { blocking myosin light chain } \\
\text { phosphorylation }\end{array}$ & MSCs & $\begin{array}{l}\text { Two-dimensional: blocks neurogenesis at } 0.1 \text { to } 1 \\
\mathrm{kPa} \text {, myogenesis at } 8 \text { to } 17 \mathrm{kPa} \text {, osteogenesis at } 25 \\
\text { to } 40 \mathrm{kPa}[6]\end{array}$ \\
\hline & & & MSCs & $\begin{array}{l}\text { Three-dimensional: no obvious effect on } \\
\text { osteogenesis at } 0.2 \text { to } 59 \mathrm{kPa}[46]\end{array}$ \\
\hline \multirow[t]{2}{*}{ Y27632 } & ROCK & $\begin{array}{l}\text { Inhibits actomyosin contractility by } \\
\text { blocking the RhoA-ROCK pathway }\end{array}$ & $\begin{array}{l}\text { Mammary } \\
\text { progenitor cells }\end{array}$ & $\begin{array}{l}\text { Two-dimensional: abrogates myoepithelial cell } \\
\text { differentiation at } 4 \mathrm{kPa} \text { [60] }\end{array}$ \\
\hline & & & MSCs & $\begin{array}{l}\text { Three-dimensional: no obvious effect on } \\
\text { hypertrophic differentiation at } \sim 53.6 \mathrm{kPa} \text { [48]; no } \\
\text { obvious effect on osteogenesis at } 0.2 \sim 59 \mathrm{kPa} \text { [46] }\end{array}$ \\
\hline Calyculin A & $\begin{array}{l}\text { Myosin light chain } \\
\text { phosphatase }\end{array}$ & $\begin{array}{l}\text { Increases actomyosin contractility by } \\
\text { inhibiting the myosin light chain } \\
\text { phosphatase }\end{array}$ & $\begin{array}{l}\text { Mammary } \\
\text { progenitor cells }\end{array}$ & $\begin{array}{l}\text { Two-dimensional: increases luminal epithelial cell } \\
\text { differentiation at } 0.1 \mathrm{kPa}[60]\end{array}$ \\
\hline Cytochalasin-D & Actin filament & Inhibits actin polymerization & ASCS & $\begin{array}{l}\text { Two-dimensional: reduces the cellular area and } \\
\text { aspect ratio of cells at } 20 \text { and } 40 \mathrm{kPa} \text { and increases } \\
\text { adipogenesis at } 2 \text { to } 40 \mathrm{kPa} \text {, especially at } 2 \mathrm{kPa} \text { [61] }\end{array}$ \\
\hline Latrunculin A & $\begin{array}{l}\text { Monomeric } \\
\text { G-actin }\end{array}$ & Inhibits actin polymerization & MSCs & $\begin{array}{l}\text { Three-dimensional: increases osteogenesis at } 0.2 \text { to } \\
59 \mathrm{kPa}[46]\end{array}$ \\
\hline Colchicine & Tubulin & Inhibits microtubule formation & MSCs & $\begin{array}{l}\text { Three-dimensional: no obvious effect on } \\
\text { osteogenesis at } 0.2 \text { to } 59 \mathrm{kPa}[46]\end{array}$ \\
\hline
\end{tabular}


on stiff matrix [26]. Stress fiber-focal adhesion complexes generate forces against the ECM, causing morphological change, migration and differentiation of the cell [30]. Furthermore, vimentin intermediate filaments, which are known to directly interact with actin, integrins, and their associated focal adhesions, may also act as mechanosensing elements as they become more punctate with increasing stiffness; and as vinculins, this punctate structure disappears when integrin-ECM binding is blocked [26].

Notably, a cell has a maximal sensitivity for a particular value of stiffness, which depends on the cytoskeletal structure and the F-actin organization; a computational model has shown that the substrate stiffness sensitivity of a cell is a bell-shaped distribution over the physiological stiffness range [31]. Based on analysis of the cell tensegrity model, cells adapt to elastically tunable substrates by translating their peak sensitivity of substrate stiffness towards a value closer to the present substrate stiffness by actin filament bundling [31]. If microtubule compression forces determined by substrate stiffness are outside the range of the sensitivity of the cells (that is, the substrate is too stiff or too soft for the cells), the cells start reinforcing or dismantling their stress fibers by increasing or decreasing actin filament bundling [31]. Therefore, MSCs cultured on matrices of medium and high stiffness present a large spreading size, well-aligned stress fibers and enhanced focal adhesion assembly, all of which contribute to a high tension state, and promote myogenic and osteogenic differentiation. Conversely, diffuse actin filament networks and small spreading area associated with a relatively poorly defined actin cytoskeleton and focal adhesion assembly are found if MSCs are grown on low stiffness matrices, leading to a neuronal phenotype [6,24] (Figure 2A).

As indicated by accumulating evidence, we speculate that the cytoskeletal-based feedback loop to reach maximal sensitivity to stiffness dominates cellular mechanotransduction. According to a cell tensegrity model-based study [31], at the beginning of mechanosensing, the initial tension caused by actomyosin-modulated contractility not only allows adherent cells to exert traction stress on surrounding substrates, but also causes microtubules inside cells to experience a resisting compression force [31]. Once cells exert traction stress and adhere to the substratum, a resultant force modulated by matrix stiffness directly acts on the microtubules as another compression force [31]. Simultaneously, the initial tension caused by acto-myosin contraction and the opposing compressive forces exerted by microtubules may also be transmitted into the nucleus through the cytoskeletal network. And these forces can be resisted by the mechanosensitive intermediate filament protein lamin-A on the basis that lamin-A levels in nuclei of stem cells correlate positively with increasing ECM stiffness
[32,33]. More importantly, lamin-A knockdown facilitates MSC differentiation towards low-stress, adipogenic phenotypes on soft substrates, whereas lamin-A overexpression promotes osteogenic phenotypes with high cytoskeletal stress or tension on stiff matrix [34]. In turn, lamin-A is likely to bind nuclear actin and influences cell contractility by regulating serum response factor (SRF) pathways, which is known to promote the expression of stress fiber-associated proteins [35,36]. Therefore, laminA could also sense the microtubule compression force and contribute to cytoskeletal organization through SRF pathways. Additionally, the microtubule compression force may be perceived and balanced by other proteins, such as vinculin and vimentin, both of which have been shown to have important roles in the mechanotransduction of mechanical stiffness [26].

Following initial focal adhesion complex formation, the resultant forces from myosin-dependent traction stress on the substratum may lead to the recruitment and activation of mechanosensitive integrin [37]. Activated integrin stimulates Rho GTPase and the downstream target protein Rho associated kinase (ROCK) to further activate MLCK. Subsequently, activated MLCK in turn mediates actin filament polymerization and actomyosin-driven contraction to generate proper cytoskeleton tension, which modulates the development and maturation of focal adhesions [37,38]. Alternatively, mechanosensitive ion channels on the cell membrane may form a molecular complex with stress fibers and focal adhesions, and convert substrate stiffness into electrical and calcium signals followed by the phosphorylation of MLCK, causing the reorganization of the actin cytoskeleton [39]. Altogether, all these matrix stiffnessinduced changes in actomyosin contractility, microtubule compression force, lamin-A level, ion channels and integrin-mediated RhoA-ROCK pathways could contribute to integrin binding and cytoskeletal organization, and ultimately change cell mechnosensitivity.

\section{Nuclear lamin circuit}

The mechanosensitive lamin-A gene circuit appears to be an important modulator of diverse transcriptional pathways, including those involving retinoic acid (RA), sterol regulatory element-binding transcription factor 1 (SREBP1), SRF, and Yes-associated protein 1 (YAP1), facilitating matrix stiffness-driven differentiation of MSCs [34]. Lamin-A can suppress MSC adipogenesis on soft substrates by decreasing the nuclear level of the adipogenic transcription factor SREBP1 [34]. Furthermore, RA inhibits osteogenesis on stiff substrate, whereas an RA antagonist not only augments osteoblast differentiation but increases lamin-A levels as well [34]. Thus, it is likely that the RA pathway, which is downstream of matrix stiffness, modulates lamin-A transcription. Meanwhile, RA 


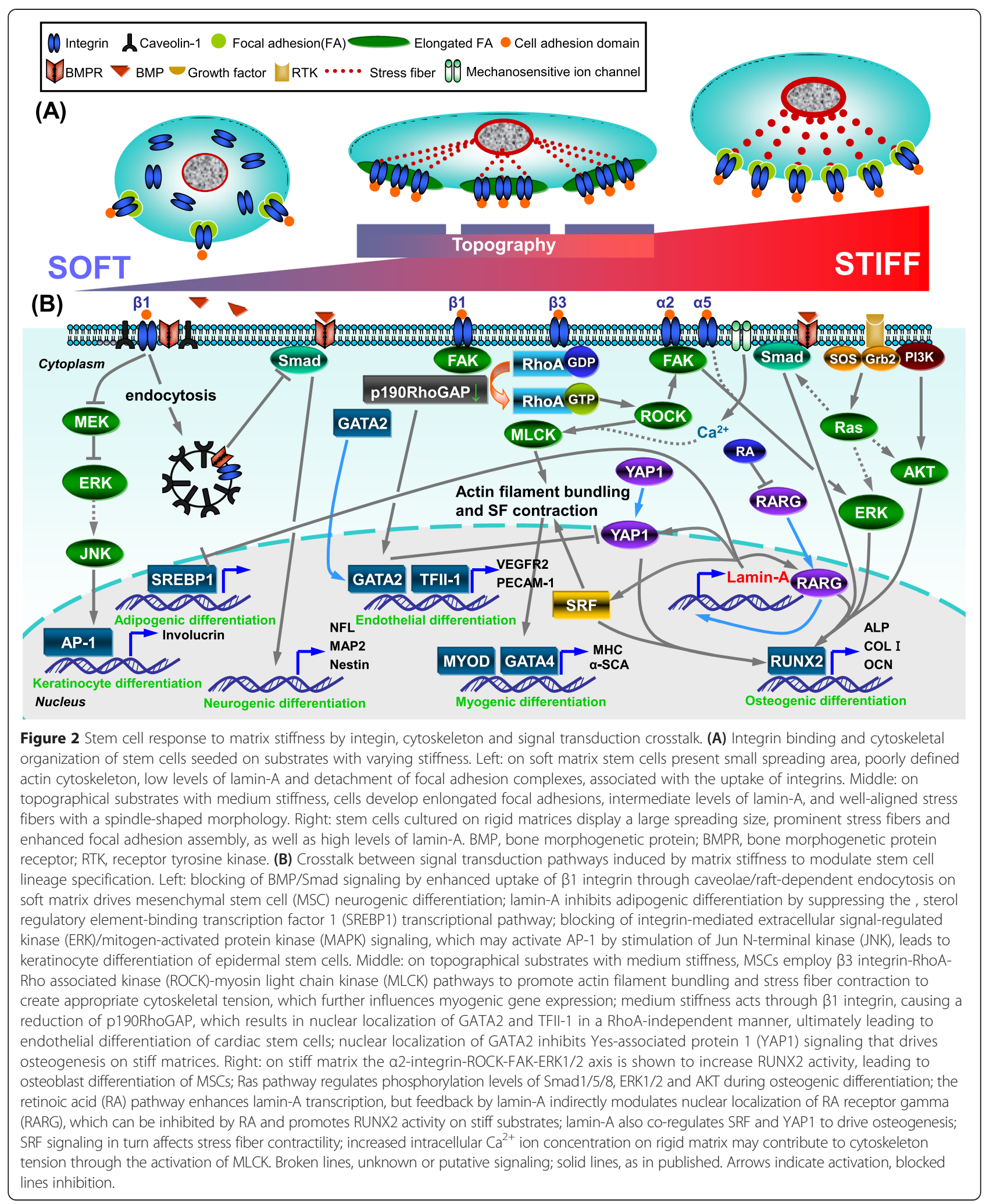

signaling or nuclear localization of retinoic acid receptor gamma (RARG) may be indirectly regulated by feedback from lamin-A, as low lamin-A favors the highest cytoplasmic levels of RARG on soft substrates and vice versa [34]. Lamin-A also influences SRF and YAP1 (a member of the Hippo signaling pathway) to drive MSC 
differentiation fate. Lamin-A overexpression in MSCs on stiff matrices is accompanied by the enrichment of YAP1 at the nuclear envelope [34]. Moreover, YAP1 concentrates at the cytoplasm during MSC adipogenesis, whereas it translocates into the nucleus during osteogenesis [40].

\section{Crosstalk network determines stem cell fate}

A complex and interconnected network of crosstalk between multiple signals triggered by matrix stiffness affects gene expression and the determination of stem cell fate (Figure 2B). MSC neurogenic differentiation on soft substrates is modulated by blocking of the BMP/Smad signaling pathway. Soft matrix promotes $\beta 1$ integrin internalization, which enhances BMPR endocytosis and further leads to the expression of neuronal genes, including microtubule associated protein 2, neurofilament protein light chain and nestin [17]. Furthermore, inhibition of extracellular signal-regulated kinase (ERK)/mitogen-activated protein kinase (MAPK) signaling regulates keratinocyte differentiation of epidermal stem cells on soft substrates [41]. It has been shown that reduced clustering of integrins ( $\beta 1$ integrin) and decreased activation of ERK/MAPK signaling may further stimulate Jun Nterminal kinase (JNK) to increase AP-1 activity, which leads to keratinocyte differentiation [41].

The endothelial cell differentiation of cardiac stem cells is induced by matrix stiffness replicating that of the myocardium (12 to $16 \mathrm{kPa}$ ) in a RhoA-independent manner, as indicated by increasing expression of platelet endothelial cell adhesion molecule- 1 and vascular endothelial growth factor receptor 2 [42]. Medium stiffness acts through $\beta 1$ integrin, causing a reduction of p190RhoGAP, and subsequently resulting in upregulation and nuclear translocalization of the endothelial-specific transcription factors GATA2 and TFII-1 independently of RhoA activation in cardiac stem cells [42]. Moreover, GATA2 expression regulated by p190RhoGAP reduces the level of YAP as part of a nuclear lamin circuit [42]. Myogenic lineage commitment of MSCs on medium substrates $(10.2 \mathrm{kPa})$ is regulated by the RhoA-ROCK-dependent cytoskeleton tension with expression of MYOD, GATA4, $\alpha$-sarcomeric actin and myosin heavy chain [12,21]. However, the signaling pathway necessary for myogenesis that is triggered by the proper cellular tension needs to be clarified in future work.

MSC osteogenesis on stiff matrices is guided by the interplay between FAK and RhoA/ROCK signaling, $\mathrm{BMP} / \mathrm{Smad}$ and Ras-mediated signaling. MSCs accommodate to ECM stiffness via an $\alpha 2$-integrin-ROCK-FAKERK1/2 axis that promotes RUNX2 activity, eventually leading to osteogenic fate [13]. The BMP/Smad signal pathway may also influence osteogenesis induced by stiff substrates, as suppression of the phosphorylation and nuclear translocation of Smad1/5/8 inhibits osteogenic gene expression of MSCs on BMP-2 mimetic peptidegrafted substrates with similar stiffness to muscle or osteoid [24]. Furthermore, matrix stiffness-driven MSC osteogenesis is also modulated by the Ras pathway associated with high phosphorylation levels of Smad1/5/8, ERK and AKT [43]. The Ras-mediated signaling acts as a master switch in signaling transduction since it orchestrates the activity of multiple signaling molecules, including ERK, phosphatidylinositol-3-kinase/AKT and Smad, to regulate diverse cellular functions [44]. One of the well studied pathways that activate Ras and the subsequent cellular response is through ligand binding to a receptor tyrosine kinase, which is linked to Ras via two proteins, Grb2 and SOS. Inhibition of Ras by a dominant negative mutant results in the attenuation of Smad, ERK and $\mathrm{AKT}$, as well as a reduction in osteogenic marker expression by MSCs [43]. However, the Ras pathways are essential but not sufficient for matrix stiffnessregulated osteogenesis, as constitutively active Ras has little effect on Smad and AKT phosphorylation or the osteogenic markers [43]. Therefore, other signaling networks involved in these processes need to be studied in the future.

\section{A rapid shift from original mechanosensing to de novo cell-derived matrix sensing in three- dimensional cultures}

Most current investigations are derived from in vitro two-dimensional cell culture models that might not truly reflect the characteristics of the cells in physiological three-dimensional environments as two-dimensional cell-matrix interactions are bidirectional, while threedimensional ones are omnidirectional. Thus, it might be more suitable to study the mechanism of matrix stiffness mechanotransduction in three-dimensional studies. In fact, inside a three-dimensional microenvironment, the softness and curvature of biomaterials hinder the formation of actin stress fibers and cell spreading, and secreted factors can be highly concentrated relative to two-dimensional conditions; thus, it is not surprising that stem cells may sense ECM stiffness in a different way [45] (Figure 3). More and more researchers have shown that the effects of matrix stiffness on stem cell morphology and differentiation are different in twodimensional versus three-dimensional cultures (Table 2).

Myosin-based cytoskeletal tension is unlikely to be involved in three-dimensional mechanosensing, but integrin binding still plays an important role according to studies using pharmacological cytoskeletal inhibitors in three-dimensional culture (Table 1). In three-dimensional scaffolds with stiffness ranging from low $(0.2 \mathrm{kPa})$ to high (59 kPa), MSCs undergoing osteogenic differentiation lack pronounced actin filaments and microtubules [46], in contrast to previous reports in two-dimensional culture 


\section{(A) 2D microenvironment}

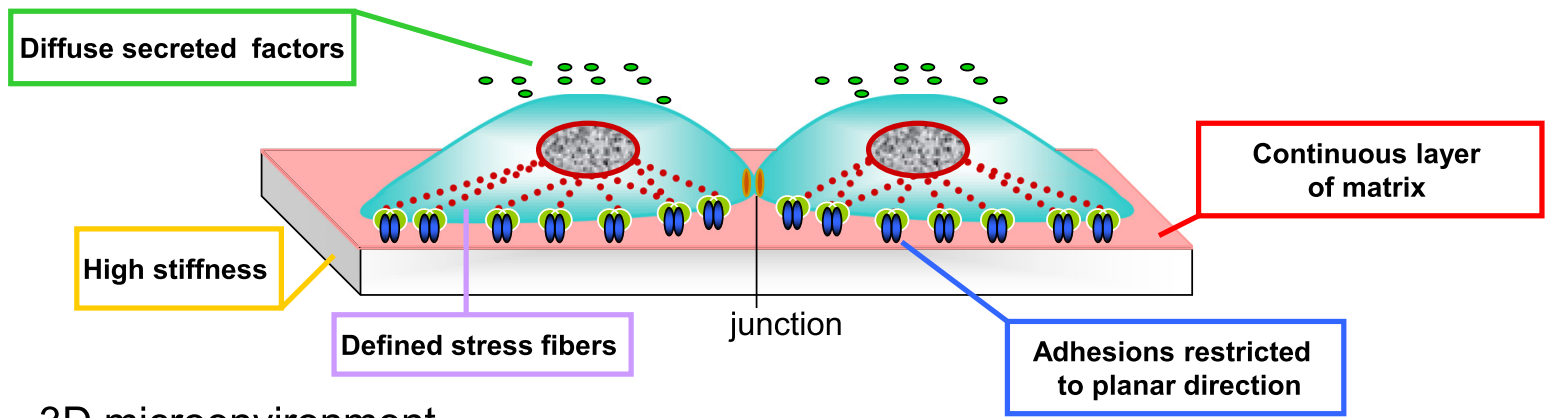

(B) 3D microenvironment to planar direction

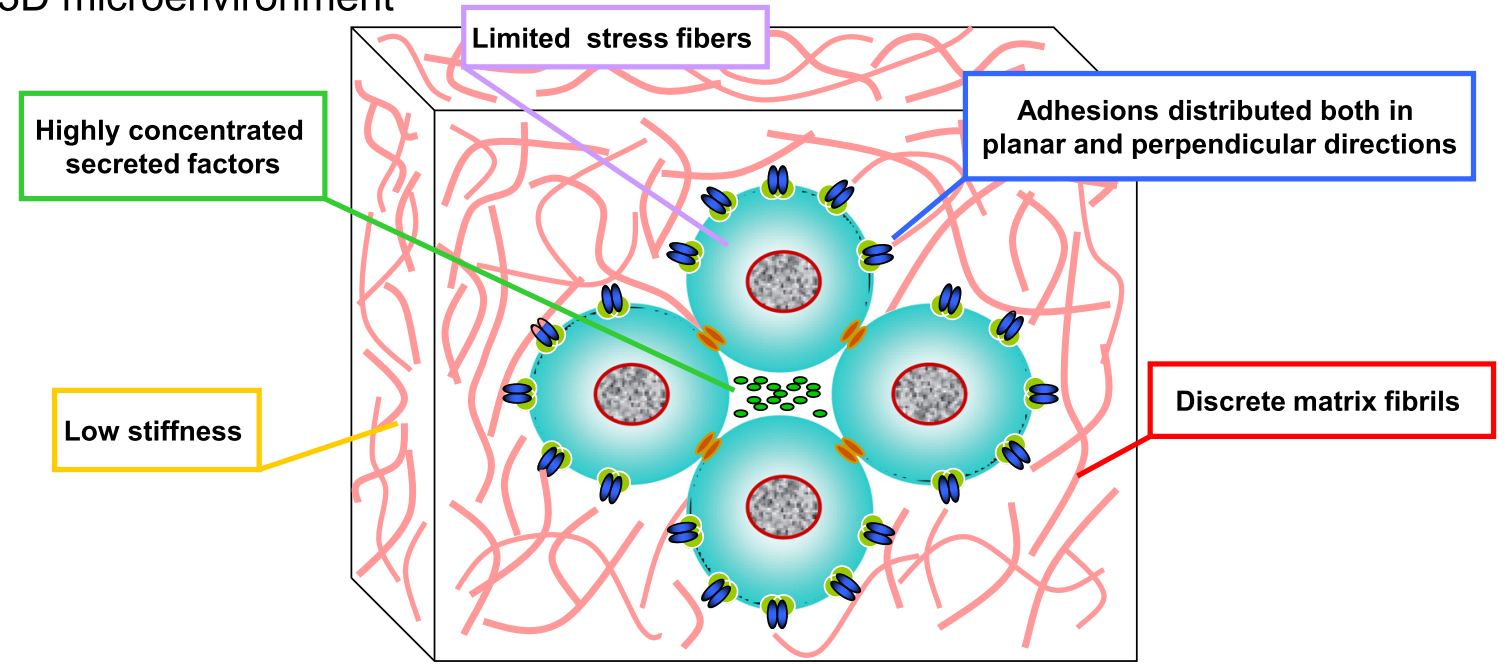

Figure 3 Discrepancy between two-dimensional and three-dimensional microenvironments. (A) Cells on a rigid two-dimensional (2D) surface coated with a continuous layer of matrix develop focal adhesions and stress fibers at the basal surface. Cell-matrix interactions are restricted to the planar direction and gradients of secreted factors are absent. (B) Inside a three-dimensional (3D) environment, cells display poorly defined stress fibers due to the softness of biomaterials. Cell adhesions are distributed both in the planar and perpendicular directions with discrete matrix fibrils, and secreted factors can be highly concentrated.

showing more well-established actin stress fibers with larger cell spreading size, along with osteogenesis on stiffer versus soft substrates [6]. When encapsulated in threedimensional scaffolds, MSCs initially express no adhesive peptides or ECM proteins, but they produce their own matrix over time, as evidenced by a great increase in fibronectin found around cells. Moreover, blocking of integrinECM interaction by RGDS inhibits MSC osteogenesis in three-dimensional scaffolds [46]. Thus, integrin binding to the de novo ECM is essential for stem cell mechanosensing in three-dimensional cultures, in accordance with previous work [47]. Nevertheless, myosin-based cytoskeletal tension and microtubules may not play a part in matrix stiffness-induced MSC differentiation in threedimensional conditions, as disruption of mechanosensing elements, including actin filament, microtubule formation, non-muscle myosin II, MLCK and ROCK, does not largely change their expression of osteogenic markers [46]. Similarly, three-dimensional hydrogels with lower stiffness (approximately $3.5 \mathrm{kPa}$ ) promote MSC chondrogenesis, whereas stiffer hydrogels (approximately $53.6 \mathrm{kPa}$ ) lead to hypertrophic differentiation with more matrix mineralization, which resembles the terminal differentiation of growthplate hypertrophic chondrocytes [48]. The greater hypertrophic differentiation may not be modulated by a force-sensing mechanotransduction mechanism because blocking of ROCK and myosin II has no obvious effect on major hypertrophic marker expression in stiff hydrogels [48]. In another study on myoblasts, cells underwent osteogenesis in medium and stiff substrates, accompanied by spherical and dot-like actin microfilaments with more micron-sized cortical protrusions that are found to enter the surrounding matrices via $\beta 1$ integrin [49]. These results again suggest that integrin binding is involved in three-dimensional mechanosensing processes.

Importantly, newly self-synthesized cell-derived ECM probably acts as a key regulator in determining stem cell 
fate. Rapid osteogenesis of myoblasts occurs on stiff (32 $\mathrm{kPa}$ ) substrates comparable to native bone tissue, as shown by highest calcium deposition and late osteogenic marker expression [49]. Interestingly, on medium substrates most cells express only early osteogenic transcripts, but they also differentiate into osteoblast-like cells at a later point in time. This phenomenon may occur because they assemble and deposit their own matrix matching the bone microenvironment [49]. Furthermore, soft hydrogels contain higher amounts of proteoglycans and collagen II, both of which are known to facilitate chondrogenesis but suppress hypertrophic differentiation and matrix calcification [50]. Additionally, more overall accumulation and uniform spatial distribution of glycosaminoglycan in soft scaffolds can bind and concentrate some hypertrophy inhibitory growth factors such as transforming growth factor $\beta \mathrm{s}$ [51]. These may be the reason why MSCs favor chondrogenic differentiation over hypertrophic differentiation in three-dimensional soft environments [48]. Extremely soft $(<0.12 \mathrm{kPa})$ three-dimensional hydrogel preferentially induces cell aggregation and osteogenic differentiation of MSCs, even without arginineglycine-aspartate (RGD) ligands [52]. In soft RGD-alginate matrices MSCs produce their own fibronectin around cell aggregates, associated with high expression of the $\alpha 5$ integrin subunit, and they are likely to effectively reinforce the hydrogel stiffness by increasing local matrix density via network contraction and fibronectin assembly [53]. Consequently, depending on cellular rearrangement, a new mechanical and biochemical microenvironment is created by local matrix stiffening, cell aggregation and $\alpha 5 \beta 1$ integrin-mediated fibronectin binding. In turn, all of these changes may control MSC commitment, as stiffer matrix and cell aggregation have a positive correlation with osteogenic differentiation [54], and $\alpha 5$ integrin signaling is known to monitor stem cell osteogenesis [55,56].

Altogether, interactions of stem cells entrapped in a three-dimensional environment with the initial matrix become less dominant over time, but newly selfsynthesized ECM where cells reside turns into a more crucial mediator of stem cell fate. Although a mechanotransduction mechanism dependent on myosin-based cytoskeletal tension and microtubules seems not to work in three-dimensional conditions, the early interaction with the intrinsic stiffness of the original matrix through integrin binding is clearly decisive. A putative shift from initial mechanosensing to de novo cell-derived matrix sensing in three-dimensional conditions may be the mechanism by which stem cells dynamically perceive their microenvironment and make fate decisions.

\section{Conclusion and future perspectives}

It is becoming increasingly clear that substrate stiffness plays a central role in determining stem cell fate in two- and three-dimensional cultures (Table 2), while the mechanisms of mechanotransduction remain unclear. Based on most results acquired from in vitro twodimensional cell culture models, cytoskeleton-based feedback loops make cells reach maximal mechanosensitivity towards matrix stiffness, and the lamin-A gene circuit plays a vital role in modulating diverse transcriptional pathways. Multiple signaling crosstalk triggered by matrix stiffness finally changes gene expression and determines stem cell fate. However, cellular mechanosensing is intricate and complex in that substrate stiffness is only one of multiple mechanical stimuli that stem cells may experience in vivo. Matrix stiffness may work in concert with other mechanical cues to regulate and coordinate stem cell behaviors, such as hydrostatic pressure and fluid shear stress [26,57]. Nevertheless, as in vivo most cells reside within a complex environment containing multiple ECM components, a medley of cell populations and mixtures of cell-secreted factors, three-dimensional cell culture models recapitulate the natural tissue environment more closely. It is highly likely that a very different regulatory mechanism of stem cell differentiation by substrate stiffness exists in three-dimensional culture. We speculate stem cells dynamically perceive their microenvironment through a shift from initial mechanosensing to newly selfsynthesized matrix sensing in a three-dimensional extracellular milieu, but the specific mechanism needs further research.

Although the value of three-dimensional cell culture has been proved repeatedly, research to identify stem cell responses to substrate stiffness in three-dimensional conditions still faces many challenges. One of the major challenges is the lack of suitable substrates with physiologically relevant properties. For instance, although thixotropic PEG-silica gels provide an inert environment for stem cell culture, which avoids the complication of biological signaling from a biologically derived matrix, their application as scaffolds are limited due to their insufficient stiffness [54]. On the contrary, silk fibroin has an inherent tendency to form stiff substrates $(>1,000$ $\mathrm{kPa}$ ), which limit its elastomeric biomaterial applications [58]. Meanwhile, transglutaminase cross-linked gelatin may be a promising biomaterial due to its mechanical stiffness $(2.5$ to $100 \mathrm{kPa})$, suitable for supporting cell differentiation during tissue regeneration [49]. Furthermore, nutrients and gas, which also are important variables that influence stem cell fates in threedimensional conditions, diffuse freely through these gels, regardless of the gel porosity or stiffness. However, although it is possible to modify the substrate stiffness by varying the degree of hydrogel cross-linking or its composition, it is impossible to eliminate the discrepancies in surface chemistry, topography and porosity. Recently, electrospinning has been utilized to create mechanically 
distinct scaffolds that have identical surface chemistries and microstructures, allowing the dissection of the effect of matrix stiffness on stem cell fate decisions in three dimensions [59].

Furthermore, cell density or cell-cell interactions might also influence the findings in three dimensions. To minimize the effects of cell density, some researchers culture cells in scaffolds for a short period until the cell density reaches approximately 70 to $80 \%$ confluence [59]. During the cellular processes the mechanical properties such as stiffness, topography and porosity may dynamically change and be distributed unevenly across the gels because of the possible degradation of gels and the endogenous ECM assembly [47]. Therefore, it is urgent to further assess the dynamic changes in mechanical properties of the substrates, and biomaterials with mechanical properties that vary across a gradient may create an ideal platform.

\section{Abbreviations}

BMP: Bone morphogenetic protein; BMPR: Bone morphogenetic protein receptor; ECM: Extracellular matrix; ERK: Extracellular signal-regulated kinase; FAK: Focal adhesion kinase; GAP: GTPase activating protein; JNK: Jun Nterminal kinase; MAPK: Mitogen-activated protein kinase; MLCK: Myosin light chain kinase; MSC: Mesenchymal stem cell; RA: Retinoic acid; RARG: Retinoic acid receptor gamma; RGD: Arginine-glycine-aspartate; RGDS: Arginineglycine-aspartate-serine; ROCK: Rho associated kinase; SREBP1: Sterol regulatory element-binding transcription factor 1; SRF: Serum response factor; YAP1: Yes-associated protein 1.

\section{Competing interests}

The authors declare that they have no competing interests.

\section{Authors' contribution}

$H L, L L$ and $Y L$ were primarily involved in drafting the review. HL prepared the figures. $L L$ and $Y L$ revised the draft manuscript and provided insights. All authors contributed to the writing of the manuscript, and read and approved the final version.

\section{Acknowledgments}

This work was supported by the State Key Development Program for Basic Research of China (grant number 2011CB606201), the National Natural Science Foundation of China (grant numbers 31150007 and 31201052), China Postdoctoral Science Foundation (grant numbers 20090450415 and 201003125), Bethune Medical Research Support Program and Advanced Interdisciplinary Innovation Project (grant number 2013101004).

\section{Author details}

${ }^{1}$ The Key Laboratory of Pathobiology, Ministry of Education, Jilin University, Changchun 130021, China. ${ }^{2}$ College of Public Health, Jilin University, Changchun 130021, China. ${ }^{3}$ College of Clinical Medicine, Jilin University, Changchun 130021, China. ${ }^{4}$ Department of Molecular Pharmacology and Experimental Therapeutics, Mayo Clinic, Rochester, MN 55902, USA.

\section{Published online: 27 May 2015}

\section{References}

1. Das M, Sundell IB, Koka PS. Adult mesenchymal stem cells and their potency in the cell-based therapy. J Stem Cells. 2013;8:1-16.

2. Eirin A, Lerman LO. Mesenchymal stem cell treatment for chronic renal failure. Stem Cell Res Ther. 2014;5:83.

3. Zeng X, Couture LA. Pluripotent stem cells for Parkinson's disease: progress and challenges. Stem Cell Res Ther. 2013;4:25.

4. Conway A, Schaffer DV. Biophysical regulation of stem cell behavior within the niche. Stem Cell Res Ther. 2012;3:50.
5. Chen JC, Jacobs CR. Mechanically induced osteogenic lineage commitment of stem cells. Stem Cell Res Ther. 2013;4:107.

6. Engler AJ, Sen S, Sweeney HL, Discher DE. Matrix elasticity directs stem cell lineage specification. Cell. 2006:126:677-89.

7. Zoldan J, Karagiannis ED, Lee CY, Anderson DG, Langer R, Levenberg S. The influence of scaffold elasticity on germ layer specification of human embryonic stem cells. Biomaterials. 2011;32:9612-21.

8. Gilbert PM, Havenstrite KL, Magnusson KEG, Sacco A, Leonardi NA, Kraft P, et al. Substrate elasticity regulates skeletal muscle stem cell self-renewal in culture. Science. 2010:329:1078-81.

9. Narayanan K, Lim WY, Shen J, Tan ZW, Rajendran D, Luo SC, et al. Extracellular matrix-mediated differentiation of human embryonic stem cells: differentiation to insulin-secreting beta cells. Tissue Eng A. 2014;20:424-33.

10. Wang T, Lai JH, Han LH, Tong X, Yang F. Chondrogenic differentiation of adipose-derived stromal cells in combinatorial hydrogels containing cartilage matrix proteins with decoupled mechanical stiffness. Tissue Eng A. 2014;20:2131-9.

11. Hynes RO. Integrins: versatility, modulation, and signaling in cell adhesion. Cell. 1992;69:11-25.

12. Yu HY, Lui YS, Xiong SJ, Leong WS, Wen F, Nurkahfianto $H$, et al. Insights into the role of focal adhesion modulation in myogenic differentiation of human mesenchymal stem cells. Stem Cells Dev. 2013;22:136-47.

13. Shih $Y R$, Tseng KF, Lai HY, Lin CH, Lee OK. Matrix stiffness regulation of integrin-mediated mechanotransduction during osteogenic differentiation of human mesenchymal stem cells. J Bone Miner Res. 2011;26:730-8.

14. Ross TD, Coon BG, Yun S, Baeyens N, Tanaka K, Ouyang M, et al. Integrins in mechanotransduction. Curr Opin Cell Biol. 2013;25:613-8.

15. Legate KR, Wickstrom SA, Fassler R. Genetic and cell biological analysis of integrin outside-in signaling. Gene Dev. 2009;23:397-418.

16. Kim C, Ye F, Ginsberg MH. Regulation of integrin activation. Annu Rev Cell Dev Biol. 2011;27:321-45.

17. Du J, Chen XF, Liang $X D$, Zhang $G Y, X u J$, He LR, et al. Integrin activation and internalization on soft ECM as a mechanism of induction of stem cell differentiation by ECM elasticity. Proc Natl Acad Sci U S A. 2011;108:9466-71.

18. Bershadsky AD, Balaban NQ, Geiger B. Adhesion-dependent cell mechanosensitivity. Annu Rev Cell Dev Biol. 2003;19:677-95.

19. Brown AEX, Discher DE. Conformational changes and signaling in cell and matrix physics. Curr Biol. 2009;19:R781-9.

20. Friedland JC, Lee MH, Boettiger D. Mechanically activated integrin switch controls alpha(5)beta(1) function. Science. 2009;323:642-4.

21. McBeath R, Pirone DM, Nelson CM, Bhadriraju K, Chen CS. Cell shape, cytoskeletal tension, and RhoA regulate stem cell lineage commitment. Dev Cell. 2004:6:483-95.

22. Cohen LA, Guan JL. Mechanisms of focal adhesion kinase regulation. Curr Cancer Drug Targets. 2005;5:629-43.

23. Michael KE, Dumbauld DW, Burns KL, Hanks SK, Garcia AJ. Focal adhesion kinase modulates cell adhesion strengthening via integrin activation. Mol Biol Cell. 2009;20:2508-19.

24. Zouani OF, Kalisky J, Ibarboure E, Durrieu MC. Effect of BMP-2 from matrices of different stiffnesses for the modulation of stem cell fate. Biomaterials. 2013;34:2157-66.

25. Kwon HJ. Chondrogenesis on sulfonate-coated hydrogels is regulated by their mechanical properties. J Mech Behav Biomed. 2013;17:337-46.

26. Steward AJ, Wagner DR, Kelly DJ. The pericellular environment regulates cytoskeletal development and the differentiation of mesenchymal stem cells and determines their response to hydrostatic pressure. Eur Cells Mater. 2013:25:167-78.

27. Inoue Y, Tsuda S, Nakagawa K, Hojo M, Adachi T. Modeling myosin-dependent rearrangement and force generation in an actomyosin network. J Theor Biol. 2011:281:65-73.

28. Geiger B, Yamada KM. Molecular architecture and function of matrix adhesions. Cold Spring Harb Perspect Biol. 2011;3:a005033.

29. Webster KD, Crow A, Fletcher DA. An AFM-based stiffness clamp for dynamic control of rigidity. PLoS One. 2011;6:e17807.

30. Mathieu PS, Loboa EG. Cytoskeletal and focal adhesion influences on mesenchymal stem cell shape, mechanical properties, and differentiation down osteogenic, adipogenic, and chondrogenic pathways. Tissue Eng B Rev. 2012;18:436-44.

31. De Santis $G$, Lennon AB, Boschetti $F$, Verhegghe $B$, Verdonck P, Prendergast PJ. How can cells sense the elasticity of a substrate? An analysis using a cell tensegrity model. Eur Cells Mater. 2011;22:202-13. 
32. Pajerowski JD, Dahl KN, Zhong FL, Sammak PJ, Discher DE. Physical plasticity of the nucleus in stem cell differentiation. Proc Natl Acad Sci U S A. 2007;104:15619-24.

33. De Vos WH, Houben F, Kamps M, Malhas A, Verheyen F, Cox J, et al. Repetitive disruptions of the nuclear envelope invoke temporary loss of cellular compartmentalization in laminopathies. Hum Mol Genet. 2011;20:4175-86.

34. Swift J, Ivanovska IL, Buxboim A, Harada T, Dingal PCDP, Pinter J, et al. Nuclear Lamin-A scales with tissue stiffness and enhances matrix-directed differentiation. Science. 2013;341:1240104.

35. Simon DN, Zastrow MS, Wilson KL. Direct actin binding to A- and B-type lamin tails and actin filament bundling by the lamin A tail. Nucleus. 2010:1:264-72.

36. Baarlink C, Wang HC, Grosse R. Nuclear actin network assembly by formins regulates the SRF coactivator MAL. Science. 2013;340:864-7.

37. Geiger B, Spatz JP, Bershadsky AD. Environmental sensing through focal adhesions. Nat Rev Mol Cell Biol. 2009;10:21-33.

38. Hirata H, Tatsumi H, Sokabe M. Mechanical forces facilitate actin polymerization at focal adhesions in a zyxin-dependent manner. J Cell Sci. 2008;121:2795-804.

39. Kobayashi T, Sokabe M. Sensing substrate rigidity by mechanosensitive ion channels with stress fibers and focal adhesions. Curr Opin Cell Biol. 2010;22:669-76.

40. Dupont S, Morsut L, Aragona M, Enzo E, Giulitti S, Cordenonsi M, et al. Role of YAP/TAZ in mechanotransduction. Nature. 2011;474:179-83.

41. Trappmann B, Gautrot JE, Connelly JT, Strange DG, Li Y, Oyen ML, et al. Extracellular-matrix tethering regulates stem-cell fate. Nat Mater. 2012;11:642-9.

42. Kshitiz, Hubbi ME, Ahn EH, Downey J, Afzal J, Kim DH, et al. Matrix rigidity controls endothelial differentiation and morphogenesis of cardiac precursors. Sci Signal. 2012;5:ra41.

43. Xue R, Li JY, Yeh Y, Yang L, Chien S. Effects of matrix elasticity and cell density on human mesenchymal stem cells differentiation. J Orthopaedic Res. 2013:31:1360-5.

44. Schindeler A, Little DG. Ras-MAPK signaling in osteogenic differentiation: friend or foe? J Bone Mineral Res. 2006;21:1331-8.

45. Adler S, Basketter D, Creton S, Pelkonen O, van Benthem J, Zuang V, et al. Alternative (non-animal) methods for cosmetics testing: current status and future prospects - 2010. Arch Toxicol. 2011;85:367-485.

46. Parekh SH, Chatterjee K, Lin-Gibson S, Moore NM, Cicerone MT, Young MF, et al. Modulus-driven differentiation of marrow stromal cells in 3D scaffolds that is independent of myosin-based cytoskeletal tension. Biomaterials. 2011;32:2256-64

47. Huebsch N, Arany PR, Mao AS, Shvartsman D, Ali OA, Bencherif SA, et al. Harnessing traction-mediated manipulation of the cell/matrix interface to control stem-cell fate. Nat Mater. 2010;9:518-26.

48. Bian LM, Hou C, Tous E, Rai R, Mauck RL, Burdick JA. The influence of hyaluronic acid hydrogel crosslinking density and macromolecular diffusivity on human MSC chondrogenesis and hypertrophy. Biomaterials. 2013;34:413-21.

49. Tan S, Fang JY, Yang Z, Nimni ME, Han B. The synergetic effect of hydrogel stiffness and growth factor on osteogenic differentiation. Biomaterials. 2014:35:5294-306.

50. Rutgers M, Saris DB, Vonk LA, van Rijen MH, Akrum V, Langeveld D, et al. Effect of collagen type I or type II on chondrogenesis by cultured human articular chondrocytes. Tissue Eng A. 2013;19:59-65.

51. Hynes RO. The extracellular matrix: not just pretty fibrils. Science. 2009;326:1216-9.

52. Maia FR, Fonseca KB, Rodrigues G, Granja PL, Barrias CC. Matrix-driven formation of mesenchymal stem cell-extracellular matrix microtissues on soft alginate hydrogels. Acta Biomater. 2014;10:3197-208.

53. Reinhart-King CA. How matrix properties control the self-assembly and maintenance of tissues. Ann Biomed Eng. 2011;39:1849-56.

54. Pek YS, Wan ACA, Ying JY. The effect of matrix stiffness on mesenchymal stem cell differentiation in a 3D thixotropic gel. Biomaterials. 2010;31:385-91.

55. Hamidouche Z, Fromigue $O$, Ringe J, Haupl T, Vaudin P, Pages JC, et al. Priming integrin alpha5 promotes human mesenchymal stromal cell osteoblast differentiation and osteogenesis. Proc Natl Acad Sci U S A. 2009;106:18587-91.
56. Gandavarapu NR, Alge DL, Anseth KS. Osteogenic differentiation of human mesenchymal stem cells on alpha 5 integrin binding peptide hydrogels is dependent on substrate elasticity. Biomater Sci. 2014;2:352-61.

57. Chen GB, LV YG, Guo P, Lin CW, Zhang XM, Yang L, et al. Matrix mechanics and fluid shear stress control stem cells fate in three dimensional microenvironment. Curr Stem Cell Res Ther. 2013;8:313-23.

58. Hu X, Park SH, Gil ES, Xia XX, Weiss AS, Kaplan DL. The influence of elasticity and surface roughness on myogenic and osteogenic-differentiation of cells on silk-elastin biomaterials. Biomaterials. 2011;32:8979-89.

59. Nam J, Johnson J, Lannutti JJ, Agarwal S. Modulation of embryonic mesenchymal progenitor cell differentiation via control over pure mechanical modulus in electrospun nanofibers. Acta Biomater. 2011;7:1516-24.

60. Lui C, Lee K, Nelson CM. Matrix compliance and RhoA direct the differentiation of mammary progenitor cells. Biomech Model Mechan. 2012;11:1241-9.

61. Young DA, Choi YS, Engler AJ, Christman KL. Stimulation of adipogenesis of adult adipose-derived stem cells using substrates that mimic the stiffness of adipose tissue. Biomaterials. 2013;34:8581-8.

62. Evans ND, Minelli C, Gentleman E, LaPointe V, Patankar SN, Kallivretaki M, et al. Substrate stiffness affects early differentiation events in embryonic stem cells. Eur Cells Mater. 2009;18:1-14.

63. Tee SY, Fu JP, Chen CS, Janmey PA. Cell shape and substrate rigidity both regulate cell stiffness. Biophys J. 2011;100:303.

64. Park JS, Chu JS, Tsou AD, Diop R, Tang Z, Wang A, et al. The effect of matrix stiffness on the differentiation of mesenchymal stem cells in response to TGF-beta. Biomaterials. 2011:32:3921-30.

65. Kothapalli CR, Kamm RD. 3D matrix microenvironment for targeted differentiation of embryonic stem cells into neural and glial lineages. Biomaterials. 2013;34:5995-6007.

66. Her GJ, Wu HC, Chen MH, Chen MY, Chang SC, Wang TW. Control of threedimensional substrate stiffness to manipulate mesenchymal stem cell fate toward neuronal or glial lineages. Acta Biomater. 2013;9:5170-80.

67. Wang LS, Boulaire J, Chan PP, Chung JE, Kurisawa M. The role of stiffness of gelatin-hydroxyphenylpropionic acid hydrogels formed by enzymemediated crosslinking on the differentiation of human mesenchymal stem cell. Biomaterials. 2010;31:8608-16.

68. Wingate K, Bonani W, Tan Y, Bryant SJ, Tan W. Compressive elasticity of three-dimensional nanofiber matrix directs mesenchymal stem cell differentiation to vascular cells with endothelial or smooth muscle cell markers. Acta Biomater. 2012;8:1440-9. 